\title{
Altering Degenerate Four-Wave Mixing and Third-Harmonic Generation in a Coupled Quantum Dot-Metallic Nanoparticle Structure with the Use of the Purcell Effect ${ }^{+}$
}

\section{Sofia Evangelou}

Citation: Evangelou, S. Altering Degenerate Four-Wave Mixing and Third-Harmonic Generation in a Coupled Quantum Dot-Metallic Nanoparticle Structure with the Use of the Purcell Effect. Mater. Proc. 2021, 4, 39. https://doi.org/10.3390/ IOCN2020-07875

Academic Editors: Ana María Díez-Pascual, Antonio Di Bartolomeo and Guanying Chen

Published: 12 November 2020

Publisher's Note: MDPI stays neutral with regard to jurisdictional claims in published maps and institutional affiliations.

Copyright: $(2020$ by the authors. Licensee MDPI, Basel, Switzerland. This article is an open access article distributed under the terms and conditions of the Creative Commons Attribution (CC BY) license (http://creativecommons.org/licenses/by/4.0/).

\author{
Department of Physics, National Technical University of Athens, 15780 Athens, Greece; \\ evangelousof@gmail.com \\ † Presented at the 2nd International Online-Conference on Nanomaterials, 15-30 November 2020; \\ Available online: https://iocn2020.sciforum.net/.
}

\begin{abstract}
The modification of the optical properties of semiconductor quantum dots near plasmonic nanostructures has attracted significant attention in recent years due to the several potential applications of the coupled nanostructures in optoelectronics, biophotonics and quantum technologies, including sensors, light harvesting, quantum information processing and quantum communication, imaging, photocatalysis, solar cells and others. One of the methods for modifying the nonlinear optical susceptibilities in quantum dots near plasmonic nanostructures uses the change of the spontaneous decay rates of quantum emitters due to the Purcell effect in a tailored nanophotonic environment. In this work, using this idea, we study the modification of the third-order nonlinear optical susceptibilities and specifically the phenomena of degenerate four-wave mixing and third-harmonic generation in a quantum dot that is coupled to a spherical metallic nanoparticle. We find that the strong alteration of the quantum dot's spontaneous decay rate near the metallic nanoparticle gives strong variation, either enhancement or suppression, of the phenomena of degenerate four-wave mixing and third-harmonic generation for different distances of the quantum dot from the surface of the metallic nanoparticle, depending on the electric dipole direction of the quantum dot. We also show that the degree of enhancement or suppression of the nonlinear optical susceptibilities differs for the studied phenomena and it is stronger for degenerate four-wave mixing than for third-harmonic generation. This work may have important potential applications in the creation of nanoscale photonic devices for various technological applications.
\end{abstract}

Keywords: Quantum dot; metallic nanoparticle; third-order optical nonlinearity; plasmonics 